\title{
Oscillopsia in middle cerebral artery dissection: a rare presentation for a rare condition
}

\begin{abstract}
We report a 46-year-old Vietnamese man who presented with acute oscillopsiaas a result of a spontaneous left middle cerebral artery (MCA) dissection. Typically, dissection of the MCA occurs in relation to head trauma. Fewer cases are thought to be purely spontaneous. Non-invasive imaging modalities led to the suspicion of an intracranial dissection that was further confirmed with cerebral angiography. Ultimately, the patient was managed conservatively.
\end{abstract}

Keywords: Middle cerebral artery (MCA), Arterial dissection, Pearl-and-string sign, Oscillopsia
Volume I Issue 5 - 2014

\section{Angelica A Nunez, Pramjeet Ahluwalia, Albert Cuetter, Alberto Maud, Gustavo J Rodriguez \\ Department of Neurology, Texas Tech University HSC, USA}

Correspondence: Angelica A. Nunez, Department of Neurology, Texas Tech University HSC, 48 Alberta Avenue, El Paso, Texas 7995, Texas, USA, Tel 915-545-6703, Fax 915-5456705;

Received: September 06, 2014 | Published: September 17, 2014
Abbreviations: MCA, Middle Cerebral Artery; CT, Computerized Tomography; MRI, Magnetic Resonance Imaging; ICA, Internal Carotid dissection

\section{Introduction}

Spontaneous cervical and cranial arterial dissection is a known cause of stroke in young adults. Arterial dissection is typically associated with minor head trauma, but nevertheless there may not be an inciting traumatic event that patients recall. Pathologically, the structural integrity of the arterial wall is compromised, allowing blood to collect between the different layers of the wall. Flow limitation and/or thromboembolism can therefore lead to cerebral ischemia. Dissection of the MCA is a rare cause of stroke when compared to dissection of the vertebrobasilar system or the cervical internal carotid arteries.

\section{Case report}

A 46-year-old Vietnamese man presented with a two-week history of on-and-off headache and blurred vision. The initial episode began as a sudden onset of headache of a moderate intensity, located in the frontal region. Associated with the headache there was blurred vision. This blurred vision was further described by the patient as a perception of objects vibrating or moving back and forth repeatedly. These symptoms resolved spontaneously over the following 3 days. The second event occurred 4 days prior to admission, at which time he had a recurrent headache. In addition he again had illusion of bouncing objects, which made it difficult to drive. This time his symptoms did not resolve and he sought care at an ophthalmology clinic. During the time between the two episodes there were periods of confusion, disorientation and difficulty with concentration. He denied fever, chills, nausea, vomiting or neck stiffness. Pertinent past medical history included tobacco use for about 10 years. No family history in relation to the present illness was reported.

On presentation he was afebrile, blood pressure was 105/70 $\mathrm{mmHg}$ and heart rate 82 with a regular rhythm. He was alert and fully oriented. Bedside cognition examination was unremarkable. He had no dysarthria or aphasia. Visual fields were full on tangent screen and $3 \mathrm{~mm}$ red target. Extra ocular muscles were normal and pupils were equal and reactive to light. Convergence was normal and there was neither ptosis nor nystagmus. The rest of the examination was otherwise normal except for unsteady gait.
Computerized tomography (CT) of the head revealed wedgeshaped hypodense areas in the left parietal, left superior and middle occipital gyry. Due to the sub acute nature of the clinical picture, a lumbar puncture was performed. Cerebro-spinal fluid studies showed; white blood cell=1, Proteins $=40 \mathrm{mg}$ dL, Glucose: $99 \mathrm{mg} \mathrm{dL}$. Bacterial, viral and fungal CSF studies were negative. Brain Magnetic resonance imaging (MRI) revealed multifocal cortical infarctions in association with a high-grade stenosis of the distal M1 segment of the left MCA (Figure 1). Our working diagnosis was that of ischemic lesions in the left MCA due to high-grade stenosis of the distal M1 segment of the MCA. A conventional cerebral angiogram showed $70 \%$ stenosis of the distal M1 segment of the left MCA prior to its bifurcation. Anintimal flap was noted consistent with dissection (Figure 2). The patient remained stable throughout his hospital stay with symptoms resolving by the time of discharge. During his admission he was diagnosed with diabetes (glycosylated hemoglobin of 9.5), and hyperlipidaemia (total cholesterol $=245$, triglycerides $=208, \mathrm{HDL}=47$, and $\mathrm{LDL}=156$ ). The management was conservative and included daily aspirin, smoking cessation, glycemic and cholesterol control.

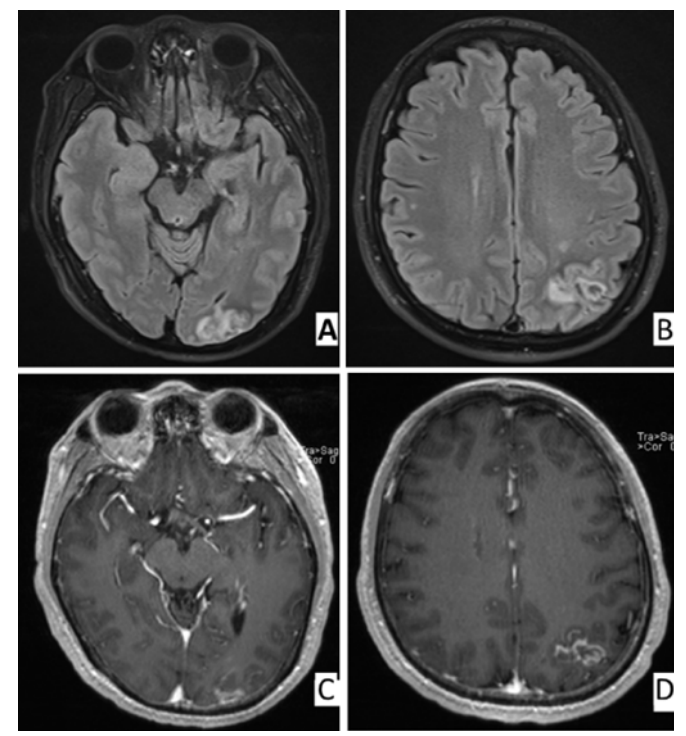

Figure I Magnetic resonance imaging of the brain axial views. FLAIR sequence demonstrating lesions in the left occipital-temporal (A) and temporal-parietal areas (B) TI sequence (+ Gadolinium) demonstrating enhancement of the above lesions ( $C \& D)$. Findings are consistent with sub acute infarcts. 


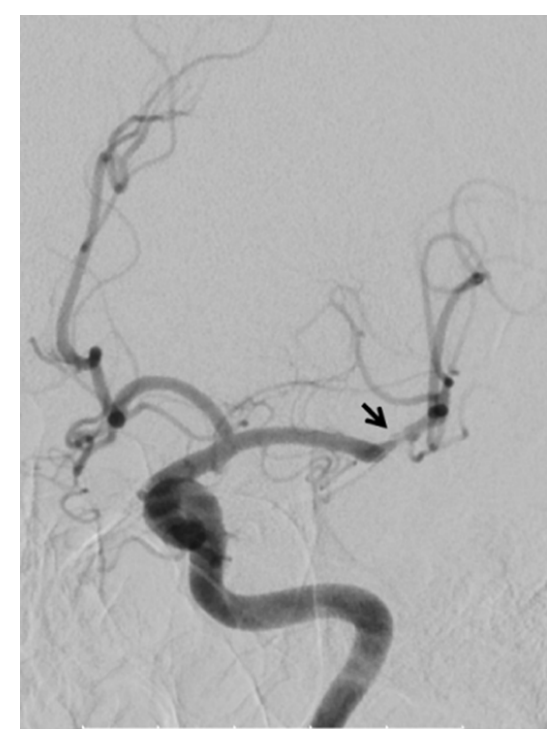

Figure 2 Digital subtraction Cerebral Angiography. Anterior-posterior view of the left internal carotid artery injection. Note the presence of an intimal flap in the distal horizontal segment (MI) of the left MCA (arrow), consistent with arterial dissection.

\section{Discussion}

We report a rare presentation of a left MCA dissection. Although headache is a common symptom, oscillopsia has not previously been reported. Oscillopsia, Illusory to-and-from movements of objects, may be a result of lesions of the geniculocalcalcarine pathway, which is a vascular domain of the MCA. Dissection of the MCA leading to stroke is a rare event in comparison to other extracranial and intracranial arterial dissections. ${ }^{1}$ Typically head trauma is associated with arterial dissection, however spontaneous dissections have also been reported. It is believed that some spontaneous dissections are in reality traumatic in origin, as trivial trauma may not be recalled by patients. Dissection of the MCA may occur as an isolated event or as an intracranial extension from an internal carotid dissection (ICA). Mobbs and Chandran have described an association of trauma and dissection of the MCA as the artery courses through the posterior margin of the sphenoid wing. ${ }^{2}$ The immediacy of the proximal segment of the MCA and the bony edge of the sphenoid wing make it vulnerable to injury even after minor trauma. ${ }^{1,2}$ In our case, the patient was working in a beauty salon when his symptoms began and did not report a provocative event. Cases of MCA dissection have also been reported as postcoital and thought to be due to acute blood pressure surges and/or trivial rotational or mechanical vessel trauma during intercourse. ${ }^{3}$

A dissection follows the direction of blood flow but the location within the arterial wall can occur between/within the three layers: the internal elastic lamina, media, and adventitia. ${ }^{4}$ Most cases of intracranial dissection present with ischemic events, however if the dissection reaches the adventitia there may be a pseudoaneurysmal formation and patients may present with a subarachnoid hemorrhage. ${ }^{5}$

In most patients with intracranial dissections of the carotid system there is an associated headache, severe and ipsilateral that immediately preceded or coexisted with the onset of the neurological deficits. ${ }^{6}$ The neurologic deficits commonly found in prior cases of MCA dissection included aphasia, hemiparesis, and dysarthria but visual disturbances such as oscillopsia have never been reported before.
The demonstration of a long tapered arterial stenosis, tapered occlusion, dissecting aneurysm (pseudoaneurysm), intimal flap, double lumen, intramural hematoma on neuroimaging strongly suggests the presence of dissection. The most common findings on a digital subtraction angiogram are; "a string sign", tapering stenosis and "flame" sign. Angiographic findings include the pearl-and-string $\operatorname{sign}^{7}$ and pathognomonic features of dissection, such as an intimal flap or double lumen are found in less than $10 \%$ of cases. A double lumenis createdby a dissection of a vessel wall where a true lumen and false lumen coexist in the same vessel. ${ }^{8}$

Management of these patients ranges from antiplatelet medications, anticoagulation, and endovascular surgery. There are currently no set guidelines. Treatment should be based on whether there is hemorrhage or ischemia, the clinical severity, and location of the lesion and its hemodynamic consequences. ${ }^{9}$ Interestingly, we found that the majority of the case reports available in the literature were young adult sand of an Asian ancestry.

\section{Conclusion}

Spontaneous dissection of the MCA should be considered as a cause of stroke in a young to middle-aged adult. Symptoms pertaining to the MCA vascular distribution include oscillopsia. Clinical suspicion and appropriate neuroimaging modalities may help elucidate the diagnosis. Different treatment approaches exist to manage intracranial dissections and treatment should be tailored to each particular patient.

\section{Acknowledgments}

None.

\section{Conflicts of Interest}

None.

All participating authors in this study declare no financial, professional or personal conflicts of interest. No grant support was received for this case report. All the above mentioned authors were involved in the preparation of this manuscript in an equal proportion.

\section{References}

1. Lee JS, Bang OY, Lee PH, et al. Two cases of spontaneous middle cerebral artery dissection causing ischemic stroke. J Neurol Sci. 2006;250(1-2):162-166.

2. Mobbs RJ, Chandran KN. Traumatic middle cerebral artery occlusion: case report and review of pathogenesis. Neurol India. 2001;49(2):158161.

3. Prabhakaran S, Krakauer JW. Multiple Reversible Episodes of subcortical ischemia following postcoital middle cerebral artery dissection. Arch Neurol. 2006;63(6):891-893.

4. Mizutani T, Kojima H, Asamoto S, et al. Pathological mechanism and three-dimensional structure of cerebral dissecting aneurysms. $J$ Neurosurg. 2001;94(5):712-717.

5. Yonas H, Agamanolis D, Takaoka T, et al. Dissecting intracranial aneurysms. Surg Neurol. 1977;8(6):407-415.

6. Sharif AA, Remley KB, Clark HB. Middle cerebral artery dissection: a clinicopathologic study. Neurology. 1995;45(10):1929-1931.

7. Yakushiji Y, Haraguchi Y, Soejima S, et al. A hyperdense artery sign and middle cerebral artery dissection; Intern Med. 2006;45(22):1319-1322.

8. Hart RG, Easton JD. Dissections of cervical and cerebral arteries. Neurol Clin. 1983;1(1):155-182.

9. Kwak HS, Hwang SB, Chung GH, et al. High-resolution magnetic resonance imaging of symptomatic middle cerebral artery dissection. $J$ Stroke Cerebrovas. 2013;23(3):550-553. 